\title{
Application Of Aboodh Transform For Solving First Order Constant Coefficients Complex Equation
}

\author{
Mohamed Elarabi Benattia ${ }^{* 1}$, Kacem Belghaba ${ }^{2}$ \\ ${ }^{1}$ Higher School of Economics, ${ }^{2}$ University of Oran 1 \\ ${ }^{1,2}$ Laboratory of Mathematics and its Applications (LAMAP),Ahmed Ben Bella, Algeria \\ ${ }^{1}$ mohamed.benattia74@yahoo.com, ${ }^{2}$ belghaba@yahoo.fr
}

\begin{abstract}
In this work, we investigate the Aboodh transformation method to solve first order constant coefficients complex equations. This method provides an effective and efficient way of solving a wide range of linear operator equations.
\end{abstract}

Keywords: Aboodh Transform, complex equation, Partial derivatives.

2010 MSC No: 45E05, 30G20, 30E20.

\section{Introduction}

Many Complex differential equations can be transformed into a system of partial differential equations that has two system of unknowns, two independent variants. separating real and imaginary parties[1]. Aboodh transformation method that is used in several areas of mathematics is an integral transform. We can solve linear differential equations, integral equations, integro-differential equations with the Aboodh transform $[2,3,4]$. This method can not be used for solving nonlinear differential equations because of nonlinear terms . But nonlinear differential equations can be solved using the Aboodh transformation aid with differential transform method. In this work, we study the solutions of first order constant coefficients complex equations. These equations were solved by Laplace transformation [1]. The organization of this paper is as follows. In Section 2,definitions and basic theorem are given. In Section 3, contains numerical illustrations.

\section{Basic definitions and theorems}

The Aboodh transform is a new integral transform similar to the Laplace transform and other integral transforms that are defined in the time domain $t \geq 0$, such as the Sumudu transform, the Natural transform and the Elzaki transform, respectively.

Aboodh transform is defined for functions of exponential order. We consider functions in the set $F$ defined by;

$$
F=\left\{f ( t ) : | f ( t ) | < M e ^ { - v t } , \text { if } t \in \left[0 ; \infty\left[, M, k_{1}, k_{2}>0 ; k_{1} \leq v \leq k_{2}\right\}\right.\right.
$$

\footnotetext{
${ }^{*}$ Corresponding author. Mohamed Elarabi Benattia ${ }^{1}$ mohamed.benattia74@yahoo.com
} 
For a given function in the set $F$, the constant $M$ must be finite number and $k_{1}, k_{2}$ may be finite or infinite with variable $v$ define as $k_{1} \leq v \leq k_{2}$. Then, the Aboodh integral transform denoted by the operator $A(:)$ is defined by the integral equation:

$$
A[f(t)]=\frac{1}{v} \int_{0}^{\infty} f(t) e^{-v t} d t, \quad t \geq 0, k_{1} \leq v \leq k_{2}
$$

Standard Aboodh transform for some special functions found are given below in Table 1.

\begin{tabular}{|c|c|}
\hline$f(x)$ & $A[f(x)]$ \\
\hline 1 & $\frac{1}{v^{2}}$ \\
\hline$x$ & $\frac{1}{v^{3}}$ \\
\hline$x^{n}, n \geq 1$ & $\frac{n !}{v^{n+2}}$ \\
\hline$e^{a t}$ & $\frac{1}{v^{2}-a v}$ \\
\hline $\sin (a x)$ & $\frac{1}{v\left(v^{2}+a^{2}\right)}$ \\
\hline $\cos (a x)$ & $\frac{1}{v^{2}+a^{2}}$ \\
\hline $\sinh (a x)$ & $\frac{1}{v\left(v^{2}-a^{2}\right)}$ \\
\hline$x \cosh (a x)$ & $\frac{1}{v^{2}-a^{2}}$ \\
\hline
\end{tabular}

TABLE1: Aboodh transform of some functions.

Aboodh transform of some partial derivatives in the form :

$$
\begin{aligned}
& A[u(x ; t)]=K(x ; v) \\
& (i)-A\left[\frac{\partial u(x ; t)}{\partial t}\right]=v K(x ; v)-\frac{u(x ; 0)}{v} . \\
& (i i)-A\left[\frac{\partial^{2} u(x ; t)}{\partial t^{2}}\right]=v^{2} K(x ; v)-\frac{1}{v} \frac{\partial u(x ; v)}{\partial t}-u(x ; 0) . \\
& (i i i)-A\left[\frac{\partial u(x ; t)}{\partial x}\right]=K^{\prime}(x ; v)=\frac{d K(x ; v)}{d x} . \\
& (i v)-A\left[\frac{\partial^{2} u(x ; t)}{\partial x^{2}}\right]=K^{\prime \prime}(x ; v)=\frac{d^{2} K(x ; v)}{d x^{2}} \text {. The Aboodh transform is linear, i.e., if } \alpha \text { and } \beta \text { are any constants }
\end{aligned}
$$
and $f(t)$ and $g(t)$ are functions defined over the set $F$ above, then

$$
A[\alpha f(x)]+A[\beta g(x)]=\alpha A[f(x)]+\beta A[g(x)]
$$

\subsection{Complex derivatives}

Let $w=w(z, \bar{z})$ be a complex function. Here $z=x+i y$, and $w(z, \bar{z})=p(x, y)+i . q(x, y)$. First order derivatives according to $z$ and $\bar{z}$ of $w(z, \bar{z})$ are defined as following:

$$
\begin{aligned}
& \frac{\partial w}{\partial z}=\frac{1}{2}\left(\frac{\partial w}{\partial x}-i \frac{\partial w}{\partial y}\right) \\
& \frac{\partial w}{\partial \bar{z}}=\frac{1}{2}\left(\frac{\partial w}{\partial x}+i \frac{\partial w}{\partial y}\right)
\end{aligned}
$$




\section{Solution of complex differential equations from first order which is constant coefficients}

Theorem 3.1. Let $a ; b ; c$ are real constants, $F(z ; \bar{z})$ is a polynomial of $z ; \bar{z}$ and $w=p+i q$ is a complex function. Then the real and imaginary parts of solution of

$$
\begin{gathered}
a \frac{\partial w}{\partial z}+b \frac{\partial w}{\partial \bar{z}}+c w=F(z ; \bar{z}) \\
w(x, 0)=0
\end{gathered}
$$

are

$$
\begin{gathered}
p=A^{-1}\left[\frac{(a+b) \frac{\partial}{\partial x}\left(2 A_{3}+\frac{(a-b)}{v} q(x, 0)\right)}{\triangle}\right. \\
\left.+\frac{2 c\left(\left(2 A_{3}+\frac{(a-b)}{v} q(x, 0)\right)-(a-b) v\left(2 A_{4}+\frac{(a-b)}{v} p(x, 0)\right)\right.}{\triangle}\right], \\
q=A^{-1}\left[\frac{(a+b) \frac{\partial}{\partial x}\left(2 A_{4}+\frac{(a-b)}{v} p(x, 0)\right)}{\triangle}\right. \\
\left.+\frac{(a-b)}{v} p(x, 0)\right)-(a-b) v\left(2 A_{3}+\frac{(a-b)}{v} q(x, 0)\right) \\
\triangle
\end{gathered} .
$$

Where

$$
\triangle=\left|\begin{array}{cc}
(a+b) D+2 c & v(a-b) \\
v(b-a) & (a+b) D+2 c
\end{array}\right|=((a+b) D+2 c)^{2}+(v(b-a))^{2} .
$$

and $A_{1}, A_{2}, A_{3}, A_{4}$ are Aboodh transforms of $p, q, F_{1}, F_{2}$ respectively.

Proof. We use equalities (3), (4) in equality (5), we have

$$
\frac{a}{2}\left(\frac{\partial w}{\partial x}-i \frac{\partial w}{\partial y}\right)+\frac{b}{2}\left(\frac{\partial w}{\partial x}+i \frac{\partial w}{\partial y}\right)+c w=F_{1}(x, y)+i F_{2}(x, y)
$$

If we chose $w=p+i q$ in (6), following equality is obtained

$$
\begin{gathered}
a\left(\frac{\partial p}{\partial x}+i \frac{\partial p}{\partial x}-i \frac{\partial p}{\partial y}+\frac{\partial q}{\partial y}\right)+b\left(\frac{\partial p}{\partial x}+i \frac{\partial p}{\partial x}+i \frac{\partial p}{\partial y}-\frac{\partial q}{\partial y}\right)+ \\
+2 c w=2 F_{1}(x, y)+2 i F_{2}(x, y)
\end{gathered}
$$

If (7) is separated to real and imagine parts, then following equation system is obtained

$$
\begin{aligned}
& (a+b) \frac{\partial p}{\partial x}+(a-b) \frac{\partial q}{\partial y}+2 c p=2 F_{1}(x, y) \\
& (a+b) \frac{\partial q}{\partial x}+(b-a) \frac{\partial p}{\partial y}+2 c q=2 F_{2}(x, y)
\end{aligned}
$$

If we use Aboodh transform for above (8), (9) equalities, then we get following equalities

$$
(a+b) \frac{\partial A_{1}}{\partial x}+(a-b)\left(v A_{2}-\frac{q(x, 0)}{v}\right)+2 c A_{1}=2 A_{3}
$$




$$
(a+b) \frac{\partial A_{2}}{\partial x}+(a-b)\left(v A_{1}-\frac{p(x, 0)}{v}\right)+2 c A_{2}=2 A_{4}
$$

If (10), (11) is regulate and is used Cramer rule, then equalities are obtained.

$$
\begin{gathered}
(a+b) \frac{\partial A_{1}}{\partial x}+(a-b) v A_{2}+2 c A_{1}=2 A_{3}+\frac{(a-b)}{v} q(x, 0) \\
(b-a) v A_{1}+(a+b) \frac{\partial A_{2}}{\partial x}+2 c A_{2}=2 A_{4}+\frac{(b-a)}{v} p(x, 0) \\
A_{1}=\frac{\mid \begin{array}{ll}
2 A_{3}+\frac{(a-b)}{v} q(x, 0) & (a-b) v \\
2 A_{4}+\frac{(b-a)}{v} p(x, 0) & (a+b) D+2 c \mid \\
\triangle
\end{array}}{A_{1}=\frac{(a+b) \frac{\partial}{\partial x}\left[2 A_{3}+\frac{(a-b)}{v} q(x, 0)\right]}{\triangle}+} \\
\frac{2 c\left(2 A_{3}+\frac{(a-b)}{v} q(x, 0)\right)-(a-b) v\left(2 A_{4}+\frac{(b-a)}{v} p(x, 0)\right)}{\triangle}
\end{gathered}
$$

and

$$
\begin{gathered}
A_{2}=\frac{\left|\begin{array}{cc}
(a+b) D+2 c & 2 A_{3}+\frac{(a-b)}{v} q(x, 0) \\
(b-a) v & 2 A_{4}+\frac{(b-a)}{v} p(x, 0)
\end{array}\right|}{\triangle} \\
A_{2}=\frac{(a+b) \frac{\partial}{\partial x}\left[2 A_{4}+\frac{(b-a)}{v} p(x, 0)\right]}{\triangle}+ \\
\frac{2 c\left(2 A_{4}+\frac{(b-a)}{v} p(x, 0)\right)-(b-a) v\left(2 A_{3}+\frac{(a-b)}{v} q(x, 0)\right)}{\triangle}
\end{gathered}
$$

Followings are obtained from inverse Aboodh of (12), (13)

$$
\begin{aligned}
& p(x, y)=A^{-1}\left[\frac{(a+b) \frac{\partial}{\partial x}\left[2 A_{3}+\frac{(a-b)}{v} q(x, 0)\right]}{\triangle}+\right. \\
& \left.\frac{2 c\left(2 A_{3}+\frac{(a-b)}{v} q(x, 0)\right)-(a-b) v\left(2 A_{4}+\frac{(b-a)}{v} p(x, 0)\right)}{\triangle}\right]
\end{aligned}
$$

and

$$
\begin{gathered}
q(x, y)=A^{-1}\left[\frac{(a+b) \frac{\partial}{\partial x}\left[2 A_{4}+\frac{(b-a)}{v} p(x, 0)\right]}{\triangle}+\right. \\
\left.\frac{2 c\left(2 A_{4}+\frac{(b-a)}{v} p(x, 0)\right)-(b-a) v\left(2 A_{3}+\frac{(a-b)}{v} q(x, 0)\right)}{\triangle}\right] .
\end{gathered}
$$


Example 3.2. Consider the following differential equation,

$$
3 \frac{\partial w}{\partial z}+\frac{\partial w}{\partial \bar{z}}=0
$$

with the condition

$$
w(x, 0)=x^{2}
$$

Coefficients of equation are $a=3, b=1, c=0$, and $F(x ; y)=0$. From the theorem (3.1), we have

$$
\triangle=((a+b) D+2 c)^{2}+(v(b-a))^{2}=16 D^{2}+4 v^{2},
$$

and

$$
\begin{aligned}
& p(x, y)=A^{-1}\left[\frac{-2 v\left(\frac{-2}{v} x^{2}\right)}{16 D^{2}+4 v^{2}}\right]=4 x^{2} A^{-1}\left[\frac{1}{16 D^{2}+4 v^{2}}\right] \\
= & x^{2} A^{-1}\left[\frac{1}{v^{2}} \cdot \frac{1}{\left(\frac{16 D^{2}}{4 v^{2}}+1\right)}\right]=x^{2} A^{-1}\left[\frac{1}{v^{2}} \cdot \frac{1}{\left(\left(\frac{2 D}{v}\right)^{2}+1\right)}\right] \\
= & x^{2} A^{-1}\left[\frac{1}{v^{2}} \cdot\left(1-\left(\frac{2 D}{v}\right)^{2}\right)\right]=A^{-1}\left[\frac{x^{2}}{v^{2}} \cdot\left(1-\left(\frac{2}{v}\right)^{2}\right)\right] \\
= & A^{-1}\left(\frac{x^{2}}{v^{2}}-\frac{8}{v^{4}}\right)=x^{2}\left(1-\frac{y^{2}}{4}\right)=x^{2}-\frac{x^{2} y^{2}}{4} .
\end{aligned}
$$

And on the other hand, we have

$$
\begin{aligned}
& q(x ; y)=A^{-1}\left[\frac{4 \partial}{\partial x}\left[\frac{\frac{-2}{v} x^{2}}{16 D^{2}+4 v^{2}}\right]=-16 x A^{-1}\left[\frac{1}{v\left(16 D^{2}+4 v^{2}\right)}\right]\right. \\
& =-4 x A^{-1}\left[\frac{1}{v^{3}\left(1+\frac{16 D^{2}}{4 v^{2}}\right)}\right]=-4 x A^{-1}\left[\frac{1}{v^{3}\left(1+\left(\frac{2 D}{v}\right)^{2}\right)}\right] \\
& =-4 x A^{-1}\left[\frac{1}{v^{3}} \cdot\left(1-\left(\frac{2 D}{v}\right)^{2}\right)\right]=-4 A^{-1}\left[\frac{x}{v^{3}} \cdot(1)\right]=-4 x y
\end{aligned}
$$

So, we obtain

$$
w=x^{2}-\frac{x^{2} y^{2}}{4}-4 i x y .
$$

Example 3.3. Consider the following differential equation,

$$
\frac{\partial w}{\partial z}+2 \frac{\partial w}{\partial \bar{z}}=z
$$

with the condition

$$
w(x ; 0)=x
$$

Coefficients of equation are $a=1, b=2, c=0$, and $F(x ; y)=x+i y$. From the theorem (3.1), we have

$$
\triangle=((a+b) D+2 c)^{2}+(v(b-a))^{2}=9 D^{2}+v^{2},
$$

and 


$$
\begin{aligned}
& p(x, y)=A^{-1}\left[\frac{3 \frac{\partial}{\partial x}\left[\frac{2 x}{v^{2}}\right]+v\left(\frac{2}{v^{3}}+\frac{1}{v} \cdot x\right)}{9 D^{2}+v^{2}}\right]=A^{-1}\left[\frac{\frac{6}{v^{2}}+v\left(\frac{2}{v^{3}}+\frac{1}{v} \cdot x\right)}{9 D^{2}+v^{2}}\right] \\
& =A^{-1}\left[\frac{\frac{8}{v^{2}}+x}{v^{2}\left(9 D^{2}+1\right)}\right]=A^{-1}\left[\frac{\left(\frac{8}{v^{2}}+x\right)}{v^{2}\left(1+\left(\frac{3 D}{v}\right)^{2}\right)}\right] \\
& =A^{-1}\left[\frac{\left(\frac{8}{v^{2}}+x\right)}{v^{2}}\left(1-\left(\frac{3 D}{v}\right)^{2}\right)\right]=A^{-1}\left[\frac{1}{v^{2}}\left(\frac{8}{v^{2}}+x\right)\right] \\
& =A^{-1}\left[\frac{8}{v^{4}}+\frac{x}{v^{2}}\right]=4 y^{2}+x . \\
& p(x, y)=A^{-1}\left[\frac{3 \frac{\partial}{\partial x}\left[\frac{2 x}{v^{2}}\right]+v\left(\frac{2}{v^{3}}+\frac{1}{v} \cdot x\right)}{9 D^{2}+v^{2}}\right]=A^{-1}\left[\frac{\frac{6}{v^{2}}+v\left(\frac{2}{v^{3}}+\frac{1}{v} \cdot x\right)}{9 D^{2}+v^{2}}\right]
\end{aligned}
$$

And on the other hand, we have

$$
\begin{gathered}
q(x, y)=A^{-1}\left[\frac{3 \frac{\partial}{\partial x}\left[\frac{2}{v^{3}}+\frac{1}{v} \cdot x\right]-v\left(2 \frac{x}{v^{2}}\right)}{9 D^{2}+v^{2}}\right]=A^{-1}\left[\frac{3}{\frac{v}{9 D^{2}+v^{2}}}\right] \\
=A^{-1}\left[\frac{(3-2 x)}{v^{3}\left(1+\left(\frac{3 D}{v}\right)^{2}\right)}\right]=A^{-1}\left[\frac{(3-2 x)}{v^{3}}\left(1-\left(\frac{3 D}{v}\right)^{2}\right)\right] \\
=A^{-1}\left[\frac{1}{v^{3}}(3-2 x)\right]=(3-2 x) y .
\end{gathered}
$$

Finally, we have

$$
w=4 y^{2}+x+i(3-2 x) y
$$

\section{References}

[1] M. Duz, On an application of Laplace transforms, New Trends in Mathematical Sciences. NTMSCI, 5(3) 2017, $53-59$.

[2] M. ElARBi BenATtiA, K. BELGHABA, Numerical Solution for Solving Fractional Differential Equations using Shifted Chebyshev Wavelet, General Letters in Mathematics, 3(2)2017, 101-110. https://doi.org/10.31559/glm2016.3.2.3

[3] T. M. Elzaki, S. M. Elzaki and E. M.A. Hilal, Elzaki and Sumudu Transforms for Solving Some Differential Equations, Global J. Pure Appl. Math., 8(2)(2012), 167-173.

[4] T. M. Elzaki, S. M. Elzaki, Application of New Transform Elzaki Transforme to Partial Differential Equations, Global J. Pure Appl. Math., 7(1)(2011), 65-70.

[5] M.A. Mohamed, M.S. Torky, Numerical solution of nonlinear system of partial differential equations by the Laplace decomposition method and the pade approximation, American Journal of Computational Mathematics,3(3)2013, 175-184. https://doi.org/10.4236/ajcm.2013.33026

[6] L. Kexue, P. Jigen, Laplace transform and fractional differential equations, Applied Mathematics Letters, 24 (2011) 2019, 20-23. https://doi.org/10.1016/j.aml.2011.05.035 
[7] E. Yusufoglu, Numerical Solution of duffing equation by the Laplace decomposition algorithm, Applied Mathematics and Computation, 177(2)2006, 572-580. https://doi.org/10.1016/j.amc.2005.07.072 